\title{
S Orionis: A Mira-type variable with a marked period decrease
}

\author{
P. Merchán Benítez and M. Jurado Vargas \\ Departamento de Física, Facultad de Ciencias, Universidad de Extremadura, 06071 Badajoz, Spain \\ e-mail: pedromer@unex.es \\ Received 14 November 2001 / Accepted 30 January 2002

\begin{abstract}
We studied the pulsational period of the Mira star S Orionis based on visual observations that cover a total of 71 years. We found that the period decreased markedly from around 445 days to 397 days in approximately 16 years, between JD 2438000 and JD 2444000. The rate of this period variation was of the order of 0.007 day/day, too fast for the usual variations observed in most Mira variables. This result is in good agreement with the theoretical models that suggest a helium-shell flash as the cause of these large-period variations. In particular, the variation of the period and luminosity indicates that this Mira star may now be in an immediate post-primary helium-shell flash state.
\end{abstract}

Key words. stars: AGB and post-AGB - stars: individual: S Ori - methods: data analysis

\section{Introduction}

In this paper we present a study of the period variations in the Mira star S Ori. This object is listed in the GCVS (Kholopov et al. 1985) as a Mira star with a period of 414.3 days and a spectral type M6.5e-M9.5e. A mean amplitude of 4.5 mag was found for S Ori by Mennessier et al. (1997). Mira-type variables are pulsating red giant stars that exhibit large amplitudes in the visible range (above $2 \mathrm{mag}$ ), long periods (usually from 100 to 1000 days), and have masses between 0.5 and $8 M_{\odot}$. These stars lie on the Asymptotic Giant Branch (AGB) of the HertzprungRussell diagram, and represent a late stage in the evolution of stars with intermediate masses. They are evolving through the tip of the AGB on a timescale of hundreds of thousands of years and, as such, are affected by two significant processes: (1) in the interior, helium shell flashes, which cause large changes in their luminosity and period on a timescale of tens of thousands of years, and (2) in the outer layers, pulsation-enhanced mass-loss, which reduces their enveloped masses, and ultimately drives them towards the white dwarf stage.

The pulsational period of a Mira star is a very important characteristic, because it constitutes an indicator of the mass, metallicity, age and mode of pulsation of the star (Wood 1990). Small period changes from cycle to cycle are usual in this type of star, although the mean period remains constant in most cases (Isles \& Saw 1987; Percy et al. 1990). In the Mira stars, every few hundred thousand years, the helium shell suffers thermal

Send offprint requests to: M. Jurado Vargas, e-mail: mjv@unex.es instabilities known as "shell flashes" or "thermal pulses", which have consequences in the star's evolution. The period changes to be expected in Mira stars due to evolution can be calculated from evolutionary models such as those of Vassiliadis \& Wood (1983). Earlier work by Wood \& Zarro (1981) had shown that the period changes could be large during certain parts of the helium shell flash cycle, although period variations of this magnitude had been observed in only three Miras (R Hya, R Aql and W Dra). Later, Gál \& Szatmáry (1995), Merchán Benítez \& Jurado Vargas (2000) and Hawkins et al. (2001) found strong period decreases in the Mira stars T UMi, S Sex and R Cen, respectively, which were also interpreted as a change in luminosity due to a flash in the helium-burning shell.

An analysis of the observational data of the Mira star S Ori is presented here. It seems to indicate that this star may also have recently undergone a helium-shell flash. The fluctuations in the period of S Ori were treated in a general study of 391 Mira stars included in the AAVSO database (Percy \& Colivas 1999), which indicated that the variations observed in $\mathrm{S}$ Ori do not seem to be due to random or cumulative errors. Our determination of the individual periods obtained from the well-defined maxima in the observations gave further evidence for a strong period change, that could be consistent with a change in luminosity caused by a flash in the helium-burning shell.

\section{Observational data}

The observational data of S Ori were taken from various sources: AAVSO (American Association of Variable Star Observers), VSOLJ (Variable Star Observers League) 

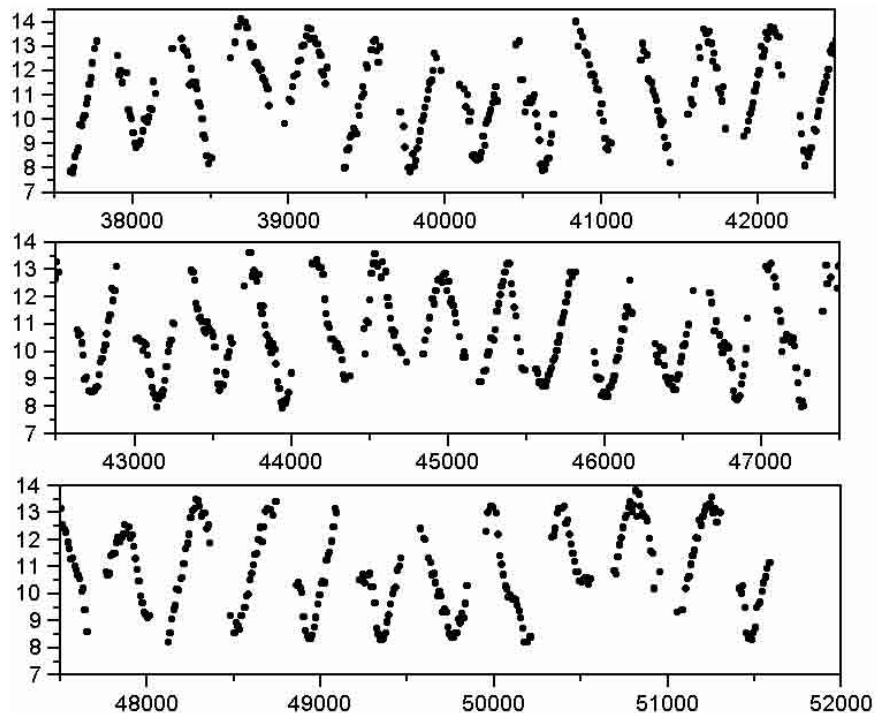

Fig. 1. Light curve of S Ori between JD 2437600 and JD 2451600.

and AFOEV (Association Francaise des Observateurs d'Étoiles Variables). A total of 8861 individual observational data were considered, corresponding to an interval of 71 years (JD 2426070-JD 2452000). The collection of data is not distributed homogeneously, because there are some incomplete cycles and many cycles with only a few or even no observations. These gaps in the observational data are especially important before JD 2436000 where the associated uncertainties are large, so that these data were not included in the detailed study of the period change. However, they will be considered in the Discussion section in order to give an overview of the temporal evolution of S Ori.

Figure 1 shows the light curve of this Mira star from JD 2437600 to JD 2451600. To facilitate visualization, each individual point in this figure represents the average of the observations in bins of 10 days. Through the 34 cycles included in this figure, one can clearly appreciate the cycle-to-cycle irregularity in the light curve of $\mathrm{S}$ Ori. The amplitude varies in the range of 3 to $6 \mathrm{mag}$, an irregularity which is common to most Mira stars

\section{Results}

The individual observations from each data source were grouped in order to obtain a more complete light curve. From this set, several well-defined maxima listed in Table 1 were determined using the Kwee-van Woerden method (Kwee \& van Woerden 1956). This table also shows the $\mathrm{O}-\mathrm{C}$ values calculated from the ephemeris reported by Kholopov et al. (1985) and the cycles elapsed from the reference epoch of this ephemeris, corresponding to the following expression:

$\operatorname{Max}=\operatorname{JD} 2443945.0+414.3 \cdot \mathrm{E}$.

Figure 2 displays these $\mathrm{O}-\mathrm{C}$ values versus Julian Date. One immediately sees the period decrease that S Ori underwent from the epoch JD 2437600 to JD 2444000, and
Table 1. Maxima obtained from the light curve of S Ori and $\mathrm{O}-\mathrm{C}$ residues relative to the ephemeris reported by Kholopov et al. (1985).

\begin{tabular}{lcc}
\hline Maximum (JD) & Cycles elapsed & O-C \\
\hline 2437589.9 & -15 & -140.6 \\
2438034.0 & -14 & -110.8 \\
2438481.0 & -13 & -78.1 \\
2439776.6 & -10 & -15.4 \\
2440205.7 & -9 & -10.6 \\
2440638.3 & -8 & 7.7 \\
2442317.5 & -4 & 29.7 \\
2442734.6 & -3 & 37.5 \\
2443144.7 & -2 & 28.3 \\
2443548.6 & -1 & 17.6 \\
2443945.0 & 0 & 0 \\
2446015.5 & 5 & -1.0 \\
2446431.3 & 6 & 0.5 \\
2446846.1 & 7 & 1.0 \\
2447257.2 & 8 & -2.2 \\
2448516.2 & 11 & 13.9 \\
2448939.1 & 12 & 22.5 \\
2449353.9 & 13 & 23.0 \\
2449760.2 & 14 & 15.0 \\
2450181.7 & 15 & 22.2 \\
2451479.4 & 18 & 77.0 \\
2451910.5 & 19 & 93.8 \\
\hline
\end{tabular}

the subsequent increase in period until the present time. However, in Mira-type stars the study of $\mathrm{O}-\mathrm{C}$ residues may be complicated by the existence of significant errors, essentially what are commonly known as "cumulative errors" and "random errors". The first are due to the accumulated difference between each observed period and the period assumed in the ephemeris, making the points drift well away from a straight line and giving the impression of real period changes. The "random errors" are due to the observational uncertainty in the estimate of maximum luminosity of long-period variables, since each maximum is common to two cycles. A wrong estimate of its position will increase the length of one cycle and decrease the length of the next.

These problems are well known in Mira-type stars and several methods have been developed to detect real changes in the mean periods (Sterne \& Campbell 1937; Lacy 1973; Isles \& Saw 1987; Lloyd 1991). Percy \& Colivas (1999) applied the methods of "contingency tables", "span test", and those proposed by Eddington \& Plakidis (1929) and Lombard \& Koen (1993) to a set of 391 Mira stars, including S Ori. Their results confirmed that S Ori suffers changes in period that were significant at a $99 \%$ confidence level. We tested the cycle lengths for change in S Ori by the contingency table method (Sterne \& Campbell 1937) in the zone where the period decrease is observed, obtaining a large value for the $\chi_{\mathrm{c}}^{2}$ parameter of 11.4. This parameter follows a $\chi^{2}$ distribution with one degree of freedom, and indicates that the change in the period of S Ori is significant at more than a $99 \%$ confidence level. This result confirms that the variations observed in the $\mathrm{O}-\mathrm{C}$ diagram 


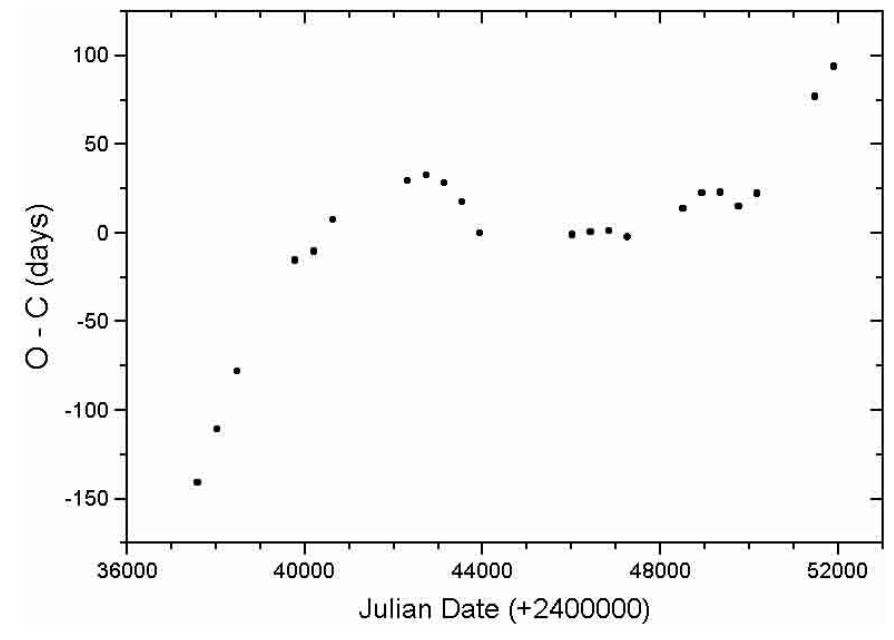

Fig. 2. O-C residues obtained from ephemeris given by Kholopov et al. (1985).

displayed in Fig. 2 are not due to random or cumulative errors.

In order to corroborate the real changes in the period of S Ori, the observations from each data source were separated into six segments, each covering about five to eight years. These segments included five to seven cycles, depending on the well-defined maxima and the number of data in the interval. In order to avoid the problems associated with the irregularly time-spaced data, the individual observations were averaged in 5 day bins prior to Fourier analysis. The mean period for each segment was then calculated. Table 2 shows the data series intervals and the resulting mean periods for each data source, and for the grouped data. These values presented an uncertainty of about 4 days in most cases.

The analysis of the first three segments, which cover a total of around 22 years, shows a clear period decrease from 455 to 415 days, approximately. In addition, the fourth and fifth segments gave a quite stable mean period of around 415 days, in perfect agreement with the period of 414.3 days given in Kholopov et al. (1985). The last interval yielded a period of around 440 days, longer than the mean period obtained in the preceding two segments, indicating a period increase in this interval.

Figure 3 plots the individual periods obtained for $\mathrm{S}$ Ori, as determined from the well-defined maxima given by the AAVSO, VSOLJ and AFOEV data bases, in addition to the maximum provided by the ephemeris of Kholopov et al. (1985). The individual cycle lengths were calculated from maximum to maximum when possible, but some mean periods had to be determined without using consecutive maxima due to the lack of homogeneity in the data distribution. It is clear that the period variations are much greater than the uncertainties associated to the individual periods. Also, one observes an abrupt change in period from JD 2438000 to JD 2444000 , followed by an increase in period until the present. An estimate of the mean rate of period decrease was obtained by performing a linear least squares fit to the data obtained between
Table 2. Data series intervals considered and the resulting mean periods.

\begin{tabular}{lcccc}
\hline Time interval (JD) & \multicolumn{5}{c}{ Mean periods } \\
& AAVSO VSOLJ AFOEV All data \\
\hline $2426070-2439000$ & 459.3 & 452.4 & - & 455.3 \\
$2439000-2442000$ & 427.8 & 425.9 & - & 431.2 \\
$2442000-2444000$ & 414.9 & 412.5 & - & 418.7 \\
$2444000-2447000$ & 415.0 & 412.1 & 413.0 & 415.3 \\
$2447000-2450000$ & 417.6 & 416.6 & 414.4 & 415.5 \\
$2450000-2451600$ & 442.5 & 441.0 & 442.0 & 437.9 \\
\hline
\end{tabular}

JD 2438000 and JD 2444000, giving a value of $\mathrm{d} P / \mathrm{d} t=$ 0.0073 day/day.

\section{Discussion}

The period decrease observed for S Ori is not typical of Mira-type variables. Although most Mira stars do not present strictly constant periods, their period variations are less than about $2 \%$ over time intervals of 10 to 50 years (Plakidis 1932; Wood 1975). Nevertheless, large and abrupt period changes have been detected in some Miras, being commonly interpreted in terms of helium flashes in the interior helium-burning shell of the star. One would expect to observe only a few percent of AGB stars in the state of helium-shell flash. In fact, large period variations had been initially observed only in the three Miras R Hya, R Aql and W Dra (Wood \& Zarro 1981; hereafter WZ), and these changes were associated with that transitional evolutionary state. Later, large continuous period decreases of around 0.006, 0.004 and 0.003 day/day were founded in T UMi (Gál \& Szatmáry 1995), S Sex (Merchán Benítez \& Jurado Vargas 2000) and R Cen (Hawkins et al. 2001), respectively, which were also interpreted as a possible change in luminosity due to a flash in the helium-burning shell of these objects. All these stars are interesting as test cases for the theoretical models that interpret such period variations as a flash in the helium-burning shell. Such a helium flash is also a possible cause of the abrupt period decrease that we observe in S Ori.

Several arguments may be made in support of a helium-flash process in S Ori. In order to give an overall vision of the stages of S Ori's evolution since 1930, the data can be structured into three well-defined parts. The first corresponds to the observations between JD 2426070 and JD 2439000, where the best estimate of the period seems to be around 455 days (Table 2). Although the data in this interval are sparse, we did not find any significant period changes, so that a certain stability can be assumed in this interval. This period stability is certainly consistent with the theoretical models of AGB stars in the phases prior to a helium flash process, as a consequence of a stable process of hydrogen-shell burning.

The second part groups the data from JD 2439000 to JD 2444000, where one clearly appreciates a marked decrease in period from 448 to 396 days, approximately, as shown in Fig. 3. In the theoretical models, when a helium 


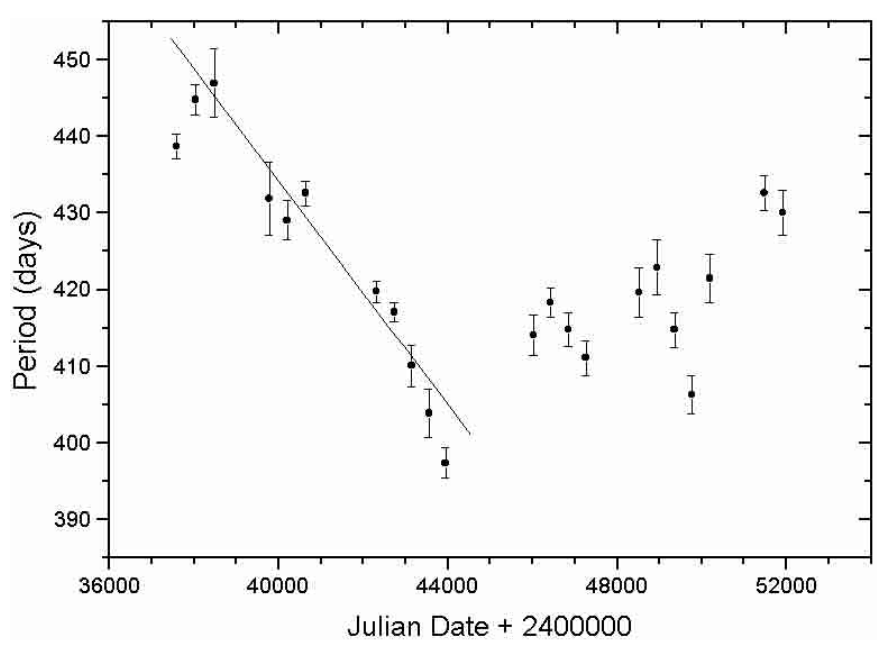

Fig. 3. Period of S Ori as a function of time. Error bars represent $2 \sigma$. Line denotes a linear fit to the data corresponding to a period decrease.

flash occurs, the hydrogen-burning shell is suddenly extinguished causing a rapid drop in the star's surface luminosity and a concomitant sharp decrease in period. This process corresponds to the beginning of the flash cycle (path from A to B in Fig. 1 of WZ) where the luminosity drop is fast. The marked decrease in period observed in $\mathrm{S}$ Ori is consistent with this part of the flash cycle where the Miras T UMi and S Sex were also placed. As predicted by the theoretical models, this luminosity drop is followed by a period increase (path from $\mathrm{B}$ to $\mathrm{C}$ in WZ). This effect is clearly detected in the third part of the data for S Ori, from around JD 2444000 until the present, as shown by the Fourier analysis (Table 2) and by the period evolution (Fig. 3). Later, another phase of the thermal pulse cycle takes place just after the maximum luminosity, caused by energy diffusion of the primary helium-flash (path from $\mathrm{C}$ to $\mathrm{D}$ in WZ), where a period decrease is also involved although less than in the first phase of the flash. R Aql and $\mathrm{R}$ Hya are examples of Mira stars located at this stage of the flash cycle. Since the period decrease observed in S Ori is abrupt and large, and is followed by a period increase, we deduce that this star must be at the beginning of the flash cycle (path from A to B to C in WZ). The theoretical models predict the more rapid period variations at this stage.

From the individual periods shown in Fig. 3 we can compare the corresponding luminosity change in S Ori with that given by the theoretical models involving helium-shell flash processes. The luminosity variation can be evaluated as a function of the period variation using equation 10 in WZ, in which the variation of luminosity for a given change of period only depends on the parameters $\beta$ and $\mathrm{b}$, and is independent of the other parameters ( $\alpha, \mathrm{C}$, a, and the stellar mass). We assumed the same values for the parameters as adopted by WZ, and which we also used in the work on S Sex (Merchán Benítez \& Jurado Vargas 2000), namely $\beta=16.67$ and $\mathrm{b}=2.0$. Comparing the luminosity change for S Ori with the curves of Fig. 3 of WZ, we obtained agreement only for stars with core mass of the order of $0.87 M_{\odot}$ or slightly greater. For lower mass stars, the luminosity drop corresponding to the path from A to B, as indicated by the theoretical model, is larger than that observed in S Ori. A determination of the S Ori mass could confirm the existence of a flash in the heliumburning shell of this star. The models also indicate that the period must finally surpass, at the point $\mathrm{C}$ of $\mathrm{WZ}$, the initial period before the flash occurred. Further observations of S Ori would then be necessary to confirm this aspect.

Within the helium flash model, another hypothesis could be considered for S Ori. The theoretical models suggest that, after the first flash and subsequent diffusion of energy, a second weaker flash occurs. However, this secondary flash usually produces a lower rate of luminosity variation than the primary, so that the period change rate observed for S Ori would be more in accordance with a primary helium-shell flash. On the other hand, some Miras, such as T Cep and S Her (WZ), show abrupt changes of period of the same magnitude expected for helium-shell flashes, only to later be reversed by a new period change similar in magnitude and timescale. This could also be the case for S Ori.

\section{Conclusion}

The analysis of visual observations of the Mira variable S Ori showed that the period of pulsation of this star has markedly decreased in the period 1963 to 1979 from 445 to around 397 days. This large period decrease is of the order of that found for other Mira stars such as R Aql, R Hya, T UMi, S Sex, and R Cen, for which the existence of a helium flash has been suggested as the cause of such variations. Therefore, the possibility that the period change observed in S Ori may be due to a change in luminosity resulting from a helium flash in the heliumburning shell cannot be discarded. Given this assumption, we suggest that $\mathrm{S}$ Ori would now be in an immediate post helium flash state, according to the models developed for stars included in the AGB. Long-term observations of this Mira are needed to corroborate the assumption of a helium flash.

Acknowledgements. Thanks are especially due to the observers and headquarters staff of AAVSO, AFOEV and VSOLJ for making their data available: without their dedication, this work would not have been possible. This research work was supported by the Junta de Extremadura (Consejería de Educación, Ciencia y Tecnología) and Fondo Social Europeo, under project IPR00A026.

\section{References}

Eddington, A. S., Plakidis, L. 1929, MNRAS, 90, 65

Gál, J., \& Szatmáry, K. 1995, A\&A, 297, 461

Hawkins, G., Mattei, J. A., \& Foster, G. 2001, PASP 113, 501 
Isles, J. E., \& Saw, D. R. B. 1987, J. Brit. Astron. Assoc., 97, 106

Kholopov, P. N., Samus, N. N., Forlov, M. S., et al. 1985, General Catalogue of Variable Stars (Nauka Publishing House, Moscow)

Kwee, K. K., \& Van Woerden, H. 1956, BAN, 12, 327

Lacy, C. H. 1973, AJ, 78, 90

Lloyd, C. 1991, J. Brit. Astron. Assoc., 101, 46

Lombard, F., \& Koen, C. J. 1993, MNRAS 263, 309

Mennessier, M. O., Boughaleb, H., \& Mattei, J.A. 1997, A\&AS, 124,143

Merchán Benítez, P., \& Jurado Vargas, M. 2000, A\&A, 353, 264

Percy, J. R., \& Colivas, T. 1999, PASP, 111, 94
Percy, J. R., Colivas, T., Sloan, W. B., \& Mattei, J. A., 1990, in Confrontation between stellar pulsation and evolution, ed. C. Cacciari, \& G. Clementini, ASP Conf. Ser., 11, 446 Plakidis, S. 1932, MNRAS, 92, 460

Sterne, T. E., \& Campbell, L. 1937, Harvard Ann., 105, 459 Vassiliadis, E., \& Wood, P. R. 1993, ApJ, 259, 198

Wood, P. R. 1975, in Multiple Periodic Variable Stars, ed. W. S. Fitch (Dordrecht: Reidel), IAU Colloq., 29, 69

Wood P. R. 1990, in From Miras to Planetary Nebulae: Which Path for Stellar Evolution?, ed. M. O. Mennessier, \& A. Omont (Gif-sur-Yvette: Éditions Frontières), 67

Wood, P. R., \& Zarro, D. M. 1981, ApJ, 247, 247 